\title{
Practical benchmarking in DEA using artificial DMUs
}

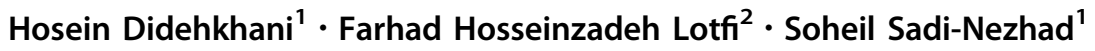

Received: 5 November 2017 / Accepted: 24 June 2018/Published online: 2 July 2018

(C) The Author(s) 2018

\begin{abstract}
Data envelopment analysis (DEA) is one of the most efficient tools for efficiency measurement which can be employed as a benchmarking method with multiple inputs and outputs. However, DEA does not provide any suggestions for improving efficient units, nor does it provide any benchmark or reference point for these efficient units. Impracticability of these benchmarks under environmental conditions is another challenge of benchmarking by DEA. The current study attempts to extend basic models for benchmarking of efficient units under practical conditions. To this end, we construct the practical production possibility set (PPPS) by employing the concept of artificial decision-making units and adding these decisionmaking units to the production possibility set (PPS) such that these artificial units satisfy all environmental constraints. Then, the theorems related to PPPS and their proofs are provided. Moreover, as a secondary result of this study, efficient units can be ranked according to their practical efficiency scores.
\end{abstract}

Keywords Artificial DMU · Benchmarking · Practical production possibility set

\section{Introduction}

Benchmarking first developed as an important business technique in computer industry in 1962. And it was in 1979 when the Xerox Company greatly improved benchmarking. In this year, the production sector of Xerox Company started assessing the technical features of its products such as its assembly line, accessories in order to compare them with those of its competitors, a practice that later came to be labeled as competitor benchmarking (Anderson and Peterson 1993). The phrase competitor benchmarking became quite popular in other sectors of organizations and

Hosein Didehkhani

h.didehkhani@gmail.com

Farhad Hosseinzadeh Lotfi

hosseinzadeh_lotfi@yahoo.com

http://www.hosseinzadeh.ir/

Soheil Sadi-Nezhad

sadinejad@hotmail.com;

https://sites.google.com/site/soheilsadinezhad

1 Department of Industrial Engineering, Science and Research Branch, Islamic Azad University, Tehran, Iran

2 Department of Mathematics, Science and Research Branch, Islamic Azad University, Tehran, Iran factories such as design, engineering and sales and marketing.

This technique was also largely used as an influential tool for surviving in contemporary competitive markets not only in manufacturing companies but also in service sectors. Pickering and Chambers (1991) presented the chief five categories of the gap analysis among the expected and levels of performance. Such categories consisted of competitor benchmarking, functional benchmarking, generic benchmarking and consumer benchmarking. Among all the different types of benchmarking, it seems that competitor benchmarking which aims at appraising the current status of a company with that of its competitors is the most prevalent kind.

Tata (2000) in another definition, benchmarking is seen as an assessment and imitation process of products, processes and systems of bests in industry. Benchmarking is also used as a tool for assessing and comparing the best methods for the improvement of business processes (Keehley et al. 1997). During recent years, it has been considered by strategic managers as one of the most influential methods of continuous improvement (Lai et al. 2011). Elmuti et al. (1997) mentioned that the advantages of benchmarking are performance evaluation, performance enhancing and enhanced learning. 
Despite this, benchmarking and an attempt in finding efficient benchmarks in business have their own challenges, which will be discussed later in this paper. The current research tries to investigate the question of benchmarking by data envelopment analysis and challenges related to it. The structure of the paper is like the following. The first section of the paper tries to give a general framework of the definition of benchmarking in competitive environments; the second section will study concepts related to the benchmarking in data envelopment analysis. The third section will deal with practical benchmarking. And the fourth section will give an illustrative example in order to show the applicability of benchmarking. And finally the last section will conclude the paper.

\section{Benchmarking in data envelopment analysis}

Different procedures and approaches have been developed to achieve efficiency measurement of economic enterprises. One of the most common and efficient models is data envelopment analysis (DEA). Data envelopment analysis is a nonparametric approach that is used in efficiency analysis. This model is an efficiency evaluation model that is capable of being used in systems that have multiple inputs and outputs. The concept of DEA was for the first time introduced by Charnes et al. (1978). After the introduction of the model, researchers developed different models for measuring efficiency evaluation.

\section{Benchmarking in DEA}

DEA offers two beneficial sets of information. The first group is related to the efficiency scores of units, and the second group is related to benchmarking information. In this way, the score for one unit is measured according to its distance from the reference units. As a result, the less the distance, the more the efficiency score of the unit under evaluation. The vector form of envelopment CCR-DEA model is as follows (Copper et al. 2007):

$\operatorname{Min} E_{\mathrm{o}}=\theta$

s.t. $\theta X_{\mathrm{o}} \geq \lambda X$

$Y_{\mathrm{o}} \leq \lambda Y$

$\lambda \geq 0$

Such that, in Model (1), $\theta=1$ indicates the associated DMU is efficient, and $\theta<1$ denotes the inefficiency of the DMU. The optimal coefficients of the envelopment model $\lambda_{j}^{*} ; j=1,2, \ldots, n$, can be used in benchmarking and deriving the reference set $R_{\mathrm{o}}=\left\{\mathrm{DMU}_{j} \mid \lambda_{j}^{*}>0\right\}$ of $\mathrm{DMU}_{\mathrm{o}}$. It is such that all reference units are efficient and dominate the efficiency of the unit under assessment. These reference units can be used as the benchmarks of inefficient units to get a higher level of efficiency. Thus, the efficient frontier introduced by DEA can also be used as the benchmarking frontier. Cook et al. (2004) developed some models to take benchmarking in DEA.

\section{Benchmarking challenges in data envelopment analysis}

Data envelopment analysis is actually a technique that is based on mathematical programming in order to find the inefficient units in a collection of decision-making units (DMUs). This means that they look for weights for the best performance of the unit under evaluation, and therefore, if there are no weights which make its performance better than the others, then it is inefficient. In vector form, it is as follows:

$$
\sim\left(\exists(U, V)>0 ; \quad \forall 1 \leq j \leq N \frac{U X_{p}}{V Y_{p}} \geq \frac{U X_{j}}{V Y_{j}}\right)
$$

where $X_{p}, Y_{p}$ are the inputs and outputs of DMU under evaluation and the $(U, V)$ are the weight vector of inputs and outputs and the $\left(X_{j}, Y_{j}\right)$ are the inputs and outputs of $j$ th DMU in observation set. In such situation, the unit studied with any weight will show the following:

$\forall(U, V)>0 ; \quad \exists j ; \quad \frac{U X_{p}}{V Y_{p}} \leq \frac{U X_{j}}{V Y_{j}}$

And thus an assessment carried out by any person, organization or model will be labeled as inefficient. But if a unit is recognized as efficient by the model, then it means:

$\exists(U, V)>0 ; \quad \forall j ; \quad \frac{U X_{p}}{V Y_{p}} \geq \frac{U X_{j}}{V Y_{j}}$

In such case, the $p$ th DMU is relatively efficient with respect to these weights; i.e., there may be other combinations of weights $(U, V)>0$ that may have lower efficiency and be inefficient. In sum, they may prove to be less efficient or inefficient when assessed by other people or models. Therefore, data envelopment analysis studies efficiency in the best of circumstances. And as such, it would be better to claim that the $p$ th DMU is in the best working condition and that we have no information regarding the efficiency of the other models that use the different weights. It can be safely claimed that the amount of efficiency calculated by data envelopment analysis is an overestimate of the amount of efficiency. Therefore, it is possible to consider an improvement in units that are efficient by DEA. Actually, it may be possible to introduce benchmark units for strongly efficient units.

The second reason for presenting a benchmark for efficient units is to stimulate motivation as well as 
competitiveness and a continual efficiency. Giving improvement suggestions to inefficient units in order to help them reach an efficient frontier, it is likely that efficient frontiers are improved by inefficient units, and if such a thing happens, efficient units that have had no improvement in their performance will become inefficient in the next period. In other words, there is no guarantee that the efficient unit of $t$ th period can prove to be as efficient as it was before.

The third reason for developing a model to generate improvement suggestion and benchmarks for efficient DMUs is related to total quality management (TQM) philosophy and continuous improvement. TQM presents a set of principles and concepts that lay emphasis on continuous improvement, consumer satisfaction and competitive benchmarking (Crosby 1984; Juran 1992). Process improvement concentrates on activities that are continuously involved with value creation in an organization. Therefore, in contemporary competitive environments, the issue of continuous improvement is vitally important. And thus, if strongly efficient units are introduced as some of the most efficient units in a group of homogeneous ones, they will still need to have help and advice in achieving a high level of efficiency.

The last challenge in benchmarking through data envelopment analysis deals with the practicality of improvement suggestions of reference benchmarks. Although these proposals and improvements have the chance to be produced in production possibility set (PPS), it is possible that the existence of different environmental constraints in the system and decision-making bodies makes it impractical and impossible to do. In other words, one of the main problems related to data envelopment analysis is the ignorance shown to environmental constraints in production possibility set. As an instance of such constraints, one can make mention of the relation that exists between teachers, employees and students of a university; in such an environment and supervisional regulations, the proportion between the teacher and the student (as the inputs of an academic system) should stay and happen within one interval. And hence such a university cannot be taken as a practical benchmark if it does not possess this virtual proportion. Other examples are those of physicians and nurses (inputs of a hospital) budget constraints of a production unit. Therefore, there is a high possibility that, in practice, we will always face such environmental and systemic constraints. Such conditions can in return damage the feasibility of improvement advice and reference benchmarks by the classic models of data envelopment analysis.

\section{Literature review}

Considering the challenges that were talked over in the reference benchmark section and suggestions for improvement related to classical data envelopment analysis, this section of the paper will attempt to shed more light on the following.

Kao (1994) presented a modified DEA model considering the allowed changes in inputs and outputs of the unit under study. The results and improvement suggestions obtained by this modified model were practical. In another study, Sowlati and Paradi (2004) presented a practical efficient frontier using the concept of artificial DMUs. Artificial units were first used by Thanasssoulis and Allen (1998). They employed the concept of artificial unit as an alternative to the weight restrictions in DEA. However, the concept used in Sowlati and Paradi (2004) was different from that used by Thanasssoulis and Allen (1998). The artificial units in Thanasssoulis and Allen (1998) had definite values for inputs and outputs, but these values were not observed in practice; rather, they were obtained based on the inputs and outputs of other units. The concept used in Sowlati and Paradi (2004) had indefinite input and output vectors, whose values are obtained from a multiplier form of CCR model.

Also the relevance of benchmarking information by Sapienza et al. (2004) was investigated. Shokrollahpour et al. (2016) proposed a new methodology for efficiency benchmarking in DEA by using ANN approach. In another research, Yi et al. (2003) studied the similarity and proximity between two benchmarks using Euclidean distances in two benchmarks. Rödder et al. (2017) proposed an interactive DEA model for measuring the return to scale of DMUs. Bogetoft and Hougaard (1998) believed that the nearest benchmark to the unit under evaluation should be used as the main example for benchmark. Post and Spronk (1999) developed the interactive model using the expert's opinions of the system. The improved model was an iterative and integrated model based on data envelopment analysis and goal programming. In this model it is tried to dwindle the distances between suggestions made-this continues until the suggestions reach an optimal level and can satisfy the experts of the systems and can also be feasible. Baek and Lee (2009) continued with the work of Halme et al. (1999) and presented a model in order to have the best and the most disciplined model using the least distance. Unlike previous models (that only presented models), it studies and measures the efficiency of the units. Ziari and Raissi (2016) developed a new model for ranking of strongly efficient DMUs in DEA by solving the infeasibility and unbounded difficulties of existing models. Esmaeilzadeh and Hadi-Vencheh (2015) proposed a 
procedure for complete ranking of DMUs in DEA. Izadikhah and Saen (2015) also developed a new model for ranking DMUs in DEA.

\section{Proposed methodology}

In this section a methodology is to be represented for a practical production possibility set (PPPS) which can guarantee the practicability of the benchmarking suggestions.

\section{Theoretical preliminaries of proposed methodology}

The main purpose of extending this model in this section is to obtain a practical benchmarking model using the concept of artificial units used by Sowlati and Paradi (2004). The model is based upon the extension of the PPS using artificial DMUs, such that the inputs and outputs of artificial units satisfy the environmental conditions and known constraints such as the inputs and outputs variation interval of each unit as well as the linear relations among inputs and outputs.

For this purpose, we assume that the model includes $N$ real $\mathrm{DMU}_{j} ; j=1,2, \ldots, N$ with known input and output vectors $\left(X_{j}, Y_{j}\right)$. The first step is to identify efficient units. To achieve this, Model (2) is used. After implementing Model (2), we can specify all the efficient units. Let us suppose that $\Omega=\left\{j \mid \mathrm{DMU}_{j}\right.$ is efficient $\}$ is the set of indexes of all efficient units and $|\Omega|=K$ to be the number of efficient units. In the envelopment CCR model under constant returns to scale, the inefficient and even weakly efficient units do not contribute to the formation of the PPS, and their elimination does not change the shape of PPS. Also inefficient units are never introduced as reference units or benchmarks to other units. So we omit them from the observation set. But as it was mentioned in the first part, the main objective of the issue is to obtain the practical efficiency and benchmarking for the strongly efficient units, since improvement of efficiency even for strongly efficient units in DEA will lead to achievement of competitive advantages in the contemporary complex and rapidly changing environments.

The modified PPS (after the elimination of inefficient and weak efficient units) should meet some practical criteria, for instance, some constraints regarding the inputs and outputs variation interval of efficient units [i.e., Sowlati and Paradi's (2004)], and the relationships among the inputs and outputs of an efficient unit. For example, the ratio of specialists to nurses in a hospital should lie within a certain interval. These relations can be obtained both through the study of the system and management opinion as well.

\section{Artificial DMU}

We introduce the artificial $\widetilde{\mathrm{DMU}_{k}}$ corresponding to the $k$ th efficient unit $k=1,2, \ldots, K$ and the matrix of $A_{k}$ as the matrix of technical coefficients of the identified constraints among the inputs and outputs of this artificial unit:

$A_{k}\left(\begin{array}{c}\tilde{X}_{k} \\ \tilde{Y}_{k}\end{array}\right) \leq b_{k}$

where, $\left(\tilde{X}_{k}, \tilde{Y}_{k}\right)$ is the vector of unknown inputs and outputs of $k$ th artificial unit. Now, we define:

$S_{k}=\left\{(X, Y) \mid A_{k}\left(\begin{array}{c}X \\ Y\end{array}\right) \leq b_{k}\right\}$

as the set of all feasible units in the set of the $k$ th constraints corresponding to the $k$ th artificial unit.

\section{Real and artificial units formation}

In this step, we form the set of all real and artificial units which should undergo benchmarking process (i.e., revised observation set). Moreover, the constraints obtained from the previous stage should be also added to PPS to construct the PPPS. In this manner, the set of (PPPS) is obtained which includes the $K$ efficient units and $K$ artificial units corresponding to those efficient units. Artificial units should satisfy the following constraints:

$$
\left(\tilde{X}_{k}, \tilde{Y}_{k}\right) \in S_{k} ; \quad k=1,2, \ldots, K
$$

\section{Benchmarking with respect to PPPS}

In this step, each of the efficient units undergoes benchmarking with respect to PPPS. The following model is an envelopment model based on (PPPS):

$E_{\mathrm{o}}^{\prime}=\operatorname{Min} \theta^{\prime}$

s.t. $\left(\theta^{\prime} X_{0}, Y_{\mathrm{o}}\right) \epsilon$ PPPS

which leads to this nonlinear model:

$E_{\mathrm{o}}^{\prime}=\operatorname{Min} \theta^{\prime}$

$\theta^{\prime} X_{0} \geq \sum_{j \in \Omega} \lambda_{j} X_{j}+\sum_{k=1}^{K} \lambda_{k}^{\prime} \tilde{X}_{k}$

$Y_{\mathrm{o}} \leq \sum_{j \in \Omega} \lambda_{j} Y_{j}+\sum_{k=1}^{K} \lambda_{k}^{\prime} \tilde{Y}_{k}$

$A_{k}\left(\begin{array}{c}\tilde{X}_{k} \\ \tilde{Y}_{k}\end{array}\right) \leq b_{k} ; \lambda_{j}, \lambda_{k}^{\prime} \geq 0 ; \quad k=1,2, \ldots, K ; \quad j \in \Omega$ 
where $\lambda_{k}^{\prime}$ is the coefficient of $k$ th artificial DMU. Considering the purpose of the statement, Model (4) is performed only for efficient units, because the conventional DEA presents some improvement suggestions and benchmarks for inefficient units. Model (4) has some differences with that of conventional model (2). Model (4) has new variables of $\lambda_{k}^{\prime}, \tilde{x}_{i k}, \tilde{y}_{r k} ; k=1,2, \ldots, K ; i=1,2, \ldots, m ; r=$ $1,2, \ldots, s$ and also new constraints. Model (4) with regard to the existence of $\lambda_{k}^{\prime} \tilde{X}_{k}$ and $\lambda_{k}^{\prime} \tilde{Y}_{k}$ is nonlinear and should be converted into linear state by using appropriate conversions.

We put $\lambda_{k}^{\prime} \tilde{y}_{r k}=q_{r k}$ and $\lambda_{k}^{\prime} \tilde{x}_{i k}=p_{i k}, i=1,2, \ldots, m ; r=$ $1,2, \ldots, s ; k=1,2, \ldots, K$, so Model (4) is converted to this linear form:

$E_{\mathrm{o}}^{\prime}=\operatorname{Min} \theta^{\prime}$

$\theta^{\prime} X_{0} \geq \sum_{j \in \Omega} \lambda_{j} X_{j}+\sum_{k=1}^{K} P_{k}$

$Y_{o} \leq \sum_{j \in \Omega} \lambda_{j} Y_{j}+\sum_{k=1}^{K} Q_{k}$

$A_{k}\left(\begin{array}{c}P_{k} \\ Q_{k}\end{array}\right) \leq \lambda_{k}^{\prime} b_{k} ; \lambda_{j}, \lambda_{k}^{\prime} \geq 0 ; k=1,2, \ldots, K ; \quad j \in \Omega$

where $P_{k}=\left(p_{1 k}, p_{2 k}, \ldots, p_{m k}\right)$ and $Q_{k}=\left(q_{1 k}, q_{2 k}, \ldots, q_{s k}\right)$. The above model is a linear model which can be easily solved by using linear programming software. In the continuation, we will deal with proving the theorems related to Model (5).

Theorem 1 Model (5) is always feasible.

Proof First we show that Model (5) is feasible for benchmarking of efficient units.

Let us assume $o \in \Omega$ and set $\lambda_{\mathrm{o}}=1, \theta^{\prime}=1$ and the rest of variables as equal to zero:

$$
\begin{aligned}
& S^{\prime}=\left(\theta ^ { \prime } = 1 , \lambda _ { 0 } = 1 ; \lambda _ { j } = 0 \text { for } \left(j \in \Omega \wedge j \neq o, \lambda^{\prime}=0,\right.\right. \\
& \quad P=0, Q=0)
\end{aligned}
$$

where $P=\left[\begin{array}{ccc}p_{11} & \cdots & p_{1 K} \\ \vdots & \ddots & \vdots \\ p_{m 1} & \cdots & p_{m K}\end{array}\right], \quad Q=\left[\begin{array}{ccc}q_{11} & \cdots & q_{1 K} \\ \vdots & \ddots & \vdots \\ q_{s 1} & \cdots & q_{s K}\end{array}\right]$ and $\lambda^{\prime}=\left(\lambda_{1}^{\prime}, \lambda_{2}^{\prime}, \ldots, \lambda_{K}^{\prime}\right)$. It is clear that $S^{\prime}$ is a feasible solution to Model (5) with objective function value $E_{\mathrm{o}}^{\prime}=\theta^{\prime}=1$.

Now assumed that $o \notin \Omega$, means the benchmarking to be performed for an inefficient unit. $S=\left(\theta^{*}, \lambda^{*}\right)$ is the optimal solution of Model (2) for deriving the efficiency of $\mathrm{DMU}_{\mathrm{o}}$. It is clear that $\theta^{*}<1$ and coefficient of none of the inefficient units $\left(\lambda_{j}^{*}\right)$ is positive; if $j \notin \Omega$, then $\lambda_{j}^{*}=0$ or in the other word, if $\lambda_{j}^{*}>0$, then $j \in \Omega$. Now we put:
$S^{\prime}=\left(\theta^{\prime}=\theta^{*}, \lambda^{*}, \lambda^{\prime}=0, P=0, Q=0\right)$

Hence, the omission of inefficient units has no impact on the possibility of Model (5), because the coefficient of none of inefficient units in Model (2) will be positive. In other words, none of the inefficient units can be part of reference units. So that, Model (5) will be feasible in the assessment and benchmarking of all units.

Theorem 2 The optimal value of Model (2) is larger or equal to the optimal value of Model (5) $\left(E_{\mathrm{o}}^{\prime} \leq E_{\mathrm{o}}\right)$

Proof Let us suppose $S=\left(\theta^{*}, \lambda^{*}\right)$ to be the optimal solution corresponding to $E_{o}$ in Model (2). Now we put.

$S^{\prime}=\left(\theta^{\prime}=\theta^{*}, \lambda_{j}^{*}>0, \lambda^{\prime}=0, P=0, Q=0\right)$

It is clear that $S^{\prime}$ is a feasible solution for Model (5) and the value of its objective function is $\theta^{*}$, so due to the fact that $E_{o}^{\prime}$ is the optimal solution to Model (5), $E_{\mathrm{o}}^{\prime} \leq \theta^{*}=E_{\mathrm{o}}$.

Corollary $1 E^{\prime} \leq 1$ for the efficient units and $E^{\prime}<1$ for inefficient units.

So, if a unit under Model (2) is inefficient, it will be inefficient by using Model (5) as well and the quantity of $E_{o}^{\prime}$ cannot be more than 1 and satisfies the definition of relative efficiency.

Eventually, after solving Model (5), we will reach to the following states:

1. $E_{\mathrm{o}}^{\prime}=E_{\mathrm{o}}=1$

In this state, the unit under benchmarking will be practically efficient too and there is no possibility for the efficiency improvement with regard to PPPS. So, maintaining the existing status is the best strategy for that. In this state, $\lambda_{k}^{\prime *}=0 ; k=1,2, \ldots, K$. So it is not possible to reach any benchmark. Because the transformations used to convert the model to linear form are not reversible.

2. $\quad E_{\mathrm{o}}^{\prime}<1$ and $E_{\mathrm{o}}=1$

which we will show that in this state, $\lambda_{k}^{\prime *}>0$ for some of artificial DMUs. So it is possible to get some of the artificial units with known vectors $\tilde{X}_{k}=\frac{P_{k}}{\lambda_{k}^{\lambda_{k}^{* k}}}, \tilde{Y}_{k}=\frac{Q_{k}}{\lambda_{k}^{* k}}$ which can be used as the practical benchmarks of that efficient unit to reach the higher level of efficiency.

Theorem 3 If $E_{\mathrm{o}}^{\prime}<1$ and $E_{\mathrm{o}}=1$, then $\exists k ; \lambda_{k}^{\prime}>0$.

Proof By contradiction, we suppose $\lambda_{k}^{* *}=0 ; k=$ $1,2, \ldots, K$ and $S^{\prime}=\left(\theta^{*}, \lambda_{j}^{*}, \lambda^{*}=0, P^{*}=0, Q^{*}=0\right)$ the optimal solution corresponding to $E_{o}^{\prime}$.

Since $\lambda^{*}=0$ for all $k=1,2, \ldots, K$ and $\lambda_{k}^{\prime} \tilde{y}_{r k}=$ $q_{r k} ; \lambda_{k}^{\prime} \tilde{x}_{i k}=p_{i k}$ implies that $P^{*}=0, Q^{*}=0$. Now we put $S=\left(\theta^{*}, \lambda_{j}^{*} ; j \in \Omega, \lambda_{j}=0 ; j \notin \Omega\right)$; it is clear that $S$ is a 
feasible solution to Model (2) whose objective function is $\theta^{\prime *}<1=\theta^{*}=E_{o}$, which is in contradiction to the optimality of $E_{o}$ to Model (2).

In summary, it can be stated that under Model (5), some of the efficient artificial units can be obtained which could be used as the practical benchmarks of efficient units. If such artificial units are impossible, then the efficient unit is practically efficient too.

\section{Illustrative example}

In this section, a numerical example is represented to illustrate the proposed methodology. In order to illustrate the PPS and Farrell's frontier using classical and proposed practical models graphically, an example with two inputs and one output is used. The example provided in this section is adopted from the literature (Esmaeilzadeh and Hadi-Vencheh 2015). Some conversions have been made on inputs and outputs to be able to present the concept of model and outlined goals in a better way. The mentioned example is related to the assessment of 7 Health Units (Hospitals). These hospitals assumed to have 2 inputs as the number of specialized physicians $\left(I_{1}\right)$ and number of nurses $\left(I_{2}\right)$ and one output as medical services to patients $(O)$. Table 1 demonstrates the input and output values of each hospital and their CCR efficiency score.

The first step in the implementation of the model is identifying efficient units. As it was described in the methodology, the model is only applied to efficient units. The mentioned CCR model evaluates seven DMUs each with two inputs and one output. Units $\mathrm{A}, \mathrm{B}$ and $\mathrm{D}$ are distinguished. Therefore, set $\Omega$ will be $\Omega=\{A, B, D\}$. Moreover, the PPS relating to these seven units and Farrell's frontier is presented in Fig. 1. As can be observed, the three units $A, B$ and $D$ lie on Farrell's frontier and are (strongly) efficient. As mentioned, inefficient units $F, C$, $E$ and $G$ do not have any role in the formation of the efficient set and Farrell's frontier, and their elimination does not change the shape of efficient set and Farrell's frontier. In the next step, we only deal with efficient units $A, B$ and $D$ for practical benchmarking since these units have the chance to be used as benchmarks for other units. The next step in implementing the model is obtaining the set of environmental and system constraints of each of the units identified as efficient. This part is the most important and also the most challenging part of the study, because all environmental and internal system constraints should be identified and designed.

Of the most important and common constraints mentioned in the studies carried out by Sowlati and Paradi (2004) and Kao (1994) are the upper and lower bounds of
Table 1 Inputs, outputs and CCR efficiency

\begin{tabular}{lllll}
\hline Unit & Doctors (I1) & Nurses (I2) & Patients (O) & CCR efficiency \\
\hline A & 18 & 151 & 210 & 1 \\
B & 19 & 131 & 205 & 1 \\
C & 25 & 160 & 215 & 0.8205 \\
D & 27 & 168 & 280 & 1 \\
E & 22 & 158 & 160 & 0.6637 \\
F & 55 & 255 & 320 & 0.7529 \\
G & 33 & 235 & 315 & 0.8739 \\
\hline
\end{tabular}

variation in inputs and outputs; such that each of the inputs or outputs can assume values only within an interval defined by management. Lower bounds of each variable are more important for inputs, since improvement suggestions indicate decrease in inputs. Therefore, specifying a lower bound for input variables makes improvement suggestions practical. However, upper bounds can also be applied, but as the model is seeking efficiency increase, it will adopt inputs at the lowest possible level. In contrast, upper bounds are important for outputs, i.e., to what extent the increase in outputs of each unit is possible. From the practical point of view, it is obvious that the output of a unit cannot be increased infinitely. Thus, following variation intervals are derived for each of the efficient units $A, B$ and $D$ by analyzing the system or hospital manager's opinions:

$\tilde{x}_{1 a} \geq 15, \tilde{x}_{1 b} \geq 15, \tilde{x}_{1 d} \geq 20$

$\tilde{x}_{2 a} \geq 145, \tilde{x}_{2 b} \geq 125, \tilde{x}_{2 d} \geq 160$

Also we have:

$\tilde{y}_{a} \leq 210, \tilde{y}_{b} \leq 205, \tilde{y}_{d} \leq 280$

For example, $\tilde{x}_{1 a} \geq 15$ means that the first input of hospital A cannot be less than 15 physicians.

Another practical constraint for a hospital can be the ratio of specialists to nurses. As there is a certain ratio of students to faculty members specified in educational centers, also there is usually a ratio of physicians to nurses specified in medical centers. For instance, it is assumed that for each efficient hospital, the ratio of specialists to nurses lies within the following interval:

$0.14 \leq \frac{\tilde{x}_{1 k}}{\tilde{x}_{2 k}} \leq 0.16$, for $k \in \Omega$

which can be rewritten as the following two linear inequalities:

$$
\begin{aligned}
& 0.14 \tilde{x}_{2 k}-\tilde{x}_{1 k} \leq 0 ; \quad k \in \Omega \\
& \tilde{x}_{1 k}-0.16 \tilde{x}_{2 k} \leq 0 ; \quad k \in \Omega
\end{aligned}
$$

Here, it should be noted that if there is no practical constraint for inputs and outputs of efficient units except the upper and lower bounds, it can be simply shown that 
Fig. 1 Farrell's frontier with CRS

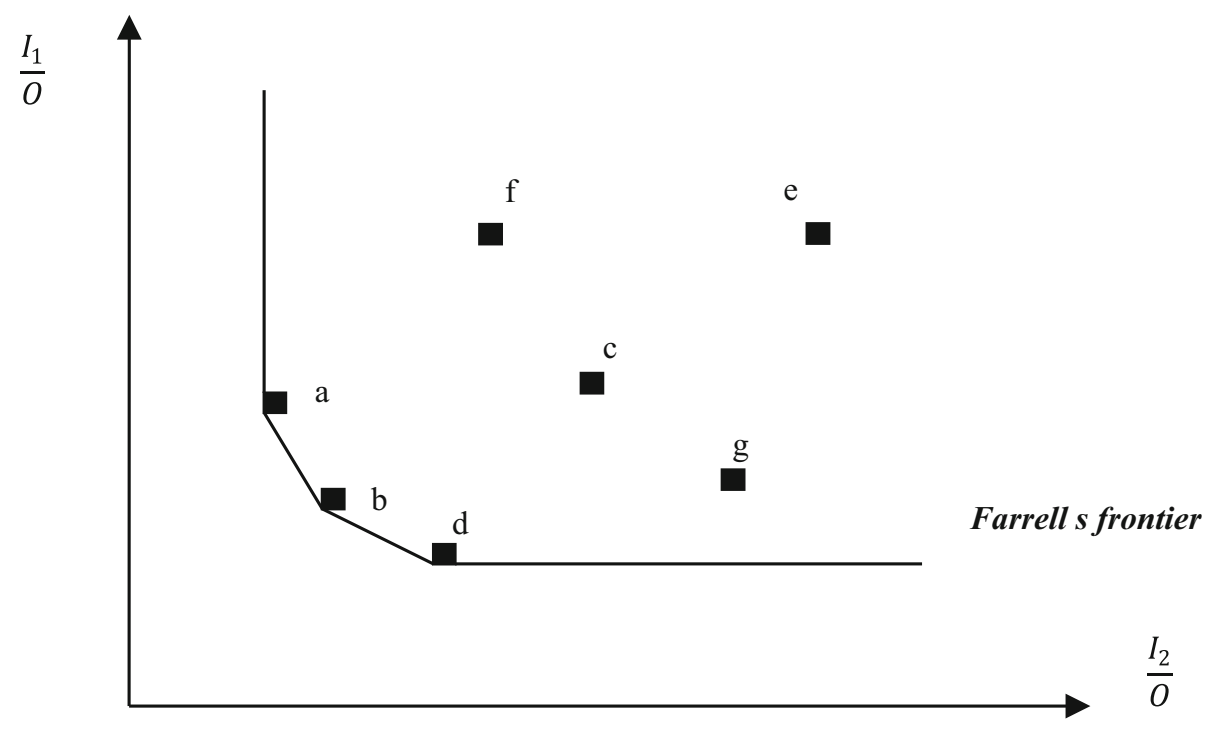

$\widetilde{\mathrm{DMU}_{k}}=\left(l_{i k}, u_{r k}\right)$ is the ideal or standard unit of the $k$ th efficient unit which dominates the $k$ th real efficient unit. Thus, pursuing the model is not sensible since an ideal benchmark for the $k$ th efficient unit can easily be introduced. However, the presence of other environmental and systemic constraints forces the inputs not to adopt their lower bounds and the outputs not to adopt their upper bounds. Hence, the unknown values of efficient benchmark units (if present) should be obtained by solving the model. Nevertheless, such constraints always exist in practical tasks.

For simplicity, it is assumed that the constraints of the system are only these two constraints. However, other constraints such as hospital budget, as a sum of the revenue paid to specialists and nurses, and other issues can also be considered.

The next step in the implementation of the model is obtaining the values $E^{\prime}$ [using Model (5)] for efficient units $A, B$ and $D$. For instance, for efficient unit $A$ and the value $E_{a}^{\prime}$, we have:

$$
\begin{aligned}
& E_{a}^{\prime}=\min \theta_{a}^{\prime} \\
& \text { s.t. } \theta_{a}^{\prime} x_{i a} \geq \sum_{j \in \Omega} \lambda_{j} x_{i j}+\sum_{k=1}^{K} p_{i k}, i=1,2 ; \\
& y_{a} \leq \sum_{j \in \Omega} \lambda_{j} y_{j}+\sum_{k=1}^{K} q_{k}, \lambda_{j}, \lambda_{k}^{\prime} \geq 0 \\
& -p_{13}+20 \lambda_{3}^{\prime} \leq 0 ; \quad-p_{11}+15 \lambda_{1}^{\prime} \leq 0 ; \quad-p_{12}+15 \lambda_{2}^{\prime} \leq 0 \\
& -p_{23}+160 \lambda_{3}^{\prime} \leq 0 ; \quad-p_{21}+145 \lambda_{1}^{\prime} \leq 0 ; \quad-p_{22}+125 \lambda_{2}^{\prime} \leq 0 \\
& q_{3}-280 \lambda_{3}^{\prime} \leq 0 ; \quad q_{1}-210 \lambda_{1}^{\prime} \leq 0 ; \quad q_{2}-205 \lambda_{2}^{\prime} \leq 0 \\
& p_{13}-0.16 p_{23} \leq 0 ; \quad p_{11}-0.16 p_{21} \leq 0 ; \quad p_{12}-0.16 p_{22} \leq 0 \\
& -p_{13}+0.14 p_{23} \leq 0 ; \quad-p_{11}+0.14 p_{21} \leq 0 ; \\
& -p_{12}+0.14 p_{22} \leq 0
\end{aligned}
$$

The second group of above constraints is those relating to upper and lower bounds of input and output variables, which have been derived using transformations; $\lambda_{k}^{\prime} \tilde{y}_{r k}=q_{r k} ; \lambda_{k}^{\prime} \tilde{x}_{i k}=p_{i k}$. Similarly, the third group of constraints is transformations of the second group of constraints of the system. The optimal solution of the above model is as follows:

$E_{a}^{\prime}=\theta_{a}^{\prime *}=0.933 ; \quad p_{13}=16.8 ; \quad p_{23}=120 ; \quad q_{3}=210 ;$ $\lambda_{3}^{*}=0.75$

Other variables are equal to zero. Since $\lambda_{3}^{\prime *}$, the transformations used for linearization of the model are reversible, and the values of $\tilde{X}_{3}$ and $\tilde{Y}_{3}$ can be obtained as follows:

$\tilde{x}_{13}=22.4 ; \quad \tilde{x}_{23}=160$ and $\tilde{y}_{3}=280$

Since $E_{a}^{\prime}<1$, the efficient unit $A$ is not practically efficient and it is possible for this unit (hospital) to consider the artificial unit with known vector $\widetilde{\mathrm{DMU}}_{3 a}=$ $(22.4,160,280)$ as its benchmark and achieve a higher level of practical efficiency. Now, by identifying the inputs and outputs of the virtual unit, the PPPS can be obtained as shown in Fig. 2.

As can be seen in Fig. 2, by adding the artificial unit $\widetilde{\mathrm{DMU}}_{3}$ all efficient units $A, B$ and $D$ lie within the frontier and are not efficient any more. Also, it can be observed in the figure that PPPS envelopes PPS, and the practical efficiency scores cannot exceed CCR efficiency scores.

Using a similar process for the efficient unit B, we have:

$E_{b}^{\prime}=\theta_{b}^{\prime *}=0.8942 ; p_{13}=16.99 ; p_{23}=117.143 ; q_{3}$

$=205 ; \lambda_{3}^{\prime *}=0.738$

and by using the reverse conversions, we have: 
Fig. 2 PPPS corresponding to the addition of $\widetilde{\mathrm{DMU}_{3}}$

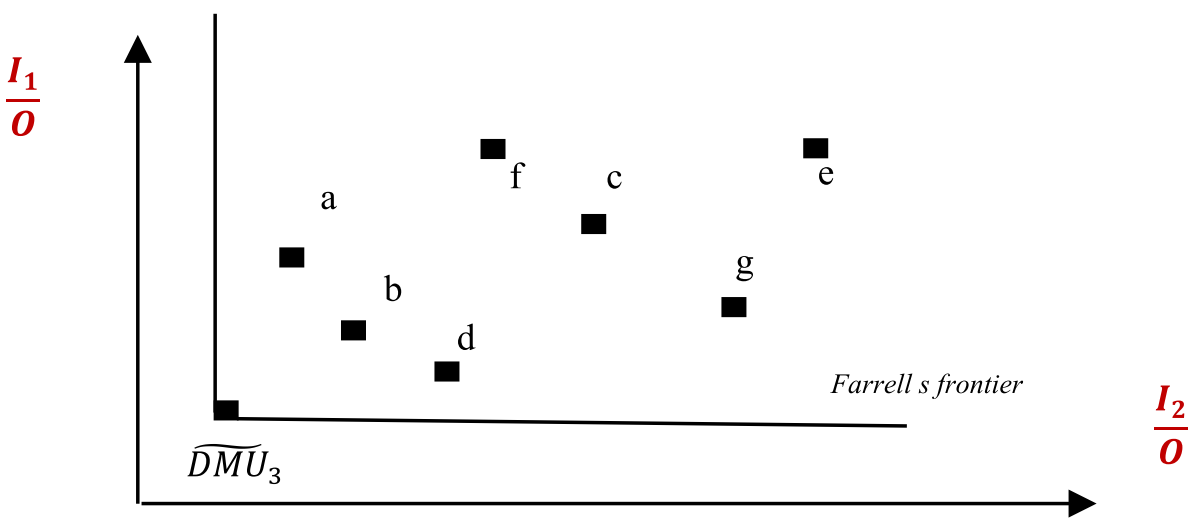

1. The modified PPS for obtaining the rank of efficient units in AP is obtained by eliminating the observed units under evaluation. Therefore, the PPS will shrink or will not change. However, the method employed in this study is based on adding some virtual units, which will certainly not lead to shrinking the PPS, i.e., the PPS will expand or will not change. Thus, we have PPS $_{\mathrm{AP}} \subseteq$ PPS and $\subseteq$ PPPS.

2. The efficiency scores obtained by AP model will result in efficiency scores greater than one, but the practical efficiency scores obtained in the current study will never be greater than one, as was proved in Theorem 1, and will be certainly smaller or equal to the CCR efficiency scores. Thus, $\theta_{\mathrm{AP}} \geq \theta_{\mathrm{CCR}}$ and $\theta_{\mathrm{CCR}} \geq \theta^{\prime}$. So, it can be observed that all processes and results obtained in this study are opposite to those of AP method, and this method can more appropriately be called a minor efficiency model.

Comparing our proposed model and AP model, we find that our model has some advantages over the latter: First, the proposed model is always feasible; secondly, efficiency scores are obtained under completely practical conditions and the definition of relative efficiency still holds, since $0 \leq \theta^{\prime} \leq 1$.

\section{Conclusion remarks}

Efficiency improvement and benchmarking have been widely considered by researchers and practitioners. Relative efficiency measurement models such as DEA, since always introduce some units efficient, do not offer any improvement suggestions for those units or any benchmark. In this paper, a practical DEA model considering the environmental and practical constraints was proposed by using the concept of artificial DMUs. The proposed nonlinear model was converted to a linear one by using the proper transformations. In comparison with the model proposed by Sowlati and Paradi (2004), the process of 
obtaining the artificial DMUs and efficiency scores of real DMUs is simultaneous. Also as the advantages of the model, the feasibility of the model and bounded efficiency scores can be mentioned. Practical efficiency scores can be used for the ranking of efficient DMUs as a proper practical ranking method, which has some advantages in comparison with AP model. Considering the vagueness and subject environment of practical constraints, uncertain approaches such as robust, fuzzy and stochastic can be applied for further research suggestions.

Open Access This article is distributed under the terms of the Creative Commons Attribution 4.0 International License (http://creative commons.org/licenses/by/4.0/), which permits unrestricted use, distribution, and reproduction in any medium, provided you give appropriate credit to the original author(s) and the source, provide a link to the Creative Commons license, and indicate if changes were made.

\section{References}

Anderson P, Peterson NC (1993) A procedure for ranking efficient units in data envelopment analysis. Manag Sci 39:1261-1264

Baek C, Lee J (2009) The relevance of DEA benchmarking information and the least-distance measure. Math Comput Model 49:265-275

Bogetoft P, Hougaard JL (1998) Efficiency evaluations based on potential (non-proportional) improvements. J Prod Anal 12:233-249

Charnes A, Cooper WW, Rhodes E (1978) Measuring the efficiency of decision making units. Eur J Oper Res 2(1):95-112

Cook WD, Seiford LM, Zhu J (2004) Models for performance benchmarking: measuring the effect of e-business activities on banking performance. Omega 32:313-322

Copper W, Seiford LM, Tone K (2007) Data envelopment analysis: a comprehensive text with models, applications, references and DEA-solver software. Springer, Berlin

Crosby PB (1984) Quality without tears. McGraw-Hill, Maidenhead

Elmuti D, Kathawala Y, Lloyed S (1997) The benchmarking process: assessing its value and limitations. Ind Manag 39(4):12-19
Esmaeilzadeh A, Hadi-Vencheh A (2015) A new method for complete ranking of DMUs. Optimization 64(5):1177-1193

Halme M, Joro T, Korhonen P, Salo S, Wallenius J (1999) A value efficiency approach to incorporating preference information in data envelopment analysis. Manag Sci 45(1):103-115

Izadikhah M, Saen RF (2015) A new data envelopment analysis method for ranking decision making units: an application in industrial parks. Expert Syst 32(5):596-608

Juran JM (1992) Juran on quality by design: the new steps for planning quality into goods and services. Free Press, New York

Kao C (1994) Efficiency improvement in data envelopment analysis. Eur J Oper Res 73:487-494

Keehley P, Medlin S, MacBride S, Longmire L (1997) Benchmarking for best practices in the public sector. Jossey Bass, California

Lai MC, Huang HC, Wang WK (2011) Designing a knowledge-based system for benchmarking: a DEA approach. Knowl-Based Syst 24:662-671

Pickering IM, Chambers S (1991) Competitive benchmarking: progress and future development. Comput Integr Manuf Syst 4:98-102

Post T, Spronk J (1999) Performance benchmarking using interactive data envelopment analysis. Eur J Oper Res 115:472-487

Rödder W, Kleine A, Dellnitz A (2017) Scaling production and improving efficiency in DEA: an interactive approach. J Ind Eng Int. https://doi.org/10.1007/s40092-017-0233-7

Sapienza HJ, Parhankangas A, Autio E (2004) Knowledge relatedness and post-spin-off growth. J Bus Ventur 19:809-829

Shokrollahpour E, Hosseinzadeh Lotfi F, Zandieh M (2016) An integrated data envelopment analysis-artificial neural network approach for benchmarking of bank branches. J Ind Eng Int 12:137-143

Sowlati T, Paradi J (2004) establishing the practical frontier in data envelopment analysis. Omega 32:261-272

Tata J (2000) Benchmarking quality management practices: US versus Costa Rica. Multinatl Bus Rev 8:37-51

Thanasssoulis E, Allen R (1998) Simulating weights restrictions in data envelopment analysis by means of unobserved DMUs. Manag Sci 44:586-594

Yi JJ, Lilja DJ, Hawkins DM (2003) A statistically rigorous approach for improving simulation methodology. In: Ninth international symposium on high performance computer architecture

Ziari S, Raissi S (2016) Ranking efficient DMUs using minimizing distance in DEA. J Ind Eng Int 12:237-242 